\title{
Stable expression and integrated hepatitis B virus genome in a human hepatoma cell line
}

\author{
L.Y. Wang ${ }^{1 *}$, Y.G. Li ${ }^{2 *}$, K. Chen ${ }^{1}$, K. Li ${ }^{1}$, J.L. Qu ${ }^{1}$, D.D. Qin ${ }^{1}$ and H. Tang ${ }^{1}$ \\ ${ }^{1}$ Key Laboratory of Molecular Biology on Infectious Diseases, \\ Ministry of Education, Second Affiliated Hospital, \\ Chongqing Medical University, Chongqing, China \\ ${ }^{2}$ Department of Forensic Medicine, Chongqing Medical University, \\ Chongqing, China \\ *These authors contributed equally to this study. \\ Corresponding author: H. Tang \\ E-mail: tanghua86162003@yahoo.com.cn
}

Genet. Mol. Res. 11 (2): 1442-1448 (2012)

Received August 25, 2011

Accepted January 13, 2012

Published May 18, 2012

DOI http://dx.doi.org/10.4238/2012.May.18.3

\begin{abstract}
HepG2.2.15 cell is a widely used cell model for studying HBV (hepatitis B virus) in vitro. In these cells, the HBV genome is integrated in several sites of HepG2 cellular DNA. These multiple copies may have some influence on the cellular processes. We constructed a new plasmid, pSEH-Flag-HBV, and transfected it into HepG2 cells, and then screened it with hygromycin. We then used ELISA, PCR, and RTPCR to detect the expression of HBV in these cell lines. A cell line that stably expressed hepatitis B e antigen ( $\mathrm{HBe} A g)$ and hepatitis B surface antigen ( $\mathrm{HBsAg}$ ) was established. Using Southern blotting analysis, we found that the HBV genome was integrated as a single copy in the cellular DNA. This cell line will be a useful alternative model for HBV studies.
\end{abstract}

Key words: Hepatitis B virus; Stable cell line; HepG2 cell; Hygromycin 


\section{INTRODUCTION}

Hepatitis B virus (HBV) is a member of the family Hepadnaviridae and causes acute and chronic liver infection in humans (Lee, 1997). HBV is difficult to study in the laboratory owing to a lack of robust models with which to research its gene expression, replication, and interaction with host genes. Although chimpanzees can be infected in vivo with HBV (Will et al., 1982) and multiplication of an HBV-like virus occurs in woodchucks, ground squirrels, and Peking ducks (Ganem et al., 1982; Halpern et al., 1984; Lindverg et al., 1985), the use of whole animals is unsuitable for detailed molecular studies. To investigate HBV replication, appropriate in vitro systems imitating the in vivo situation as closely as possible are necessary.

Many efforts have been made to establish a cell system that allows the replication of this virus. The most common of the cell lines established so far, HepG2.2.15, contains two head-totail copies of the HBV genome between the long terminal repeats of the Moloney leukemia virus (Knowles et al., 1980; Sells et al., 1987). This cell line is based on the human hepatoblastoma cell line HepG2. Another well-characterized cell line that produces stable HBV particles from a chromosomally integrated state is the HB611 cell line, which was established using Huh6-c15 cells with plasmid p3HB-neo and carries 3 tandemly arranged HBV genomes (Ochiya et al., 1989). To create the HepG2-4A5 cell line, HepG2 cells were transfected with plasmid pSPT1.2 HBV genes carrying a neomycin-resistant gene controlled by the herpes simplex thymidine kinase promoter (Weiss et al., 1996). Although these tissue culture systems have been helpful for the in vitro production of HBV, they do not allow studies of the regulation of HBV replication because some of them harbor more than one HBV genome, and at present, stable cell lines that express HBV genes have mostly been built with neomycin resistance. The lack of stable HBV-expressing cell lines has limited the study of the relationship between HBV and host genes.

We have established the cell line HepG2-H4, derived from a human hepatocellular carcinoma HepG2 cell line, through transfection with a recombinant plasmid containing 1.3fold the full-length HBV genome (genotype B). This cell line has been stably maintained, and HBV has been integrated into a single site of the cellular chromosomes. This system provides an opportunity for further study of the life cycle and biology of HBV in vitro.

\section{MATERIAL AND METHODS}

\section{Construction of the plasmid vector}

A recombinant vector, pSEH-Flag-HBV, was prepared via insertion of HBV DNA containing fragments from a HindIII digest of pcDNA3.1-HBV into the HindIII site in pSEHFlag (provided by Prof. Tongchuan He, University of Chicago, USA), a plasmid carrying the hygromycin gene. A test for the pSEH-Flag-HBV monomer was performed through HindIII digestion of mini-prep DNA. The nucleotide sequence of the DNA fragments inserted into the vector was verified with sequencing.

\section{Transfection and cell culture}

The human hepatoblastoma cell line HepG2 was maintained in $10 \%$ fetal bovine serum (GIBCO) in HyClone modified Eagle's medium at $37^{\circ} \mathrm{C}$ in an atmosphere of $5 \% \mathrm{CO}_{2}$ in 
air. The most suitable concentration of hygromycin $(450 \mu \mathrm{g} / \mathrm{mL})$ was obtained after 10 days of continued administration. HepG2 cells were then seeded on 6-well plates at a density of $1.5 \mathrm{x}$ 106/well. Transfection was accomplished with Lipofectamine 2000 reagent (Invitrogen, Carlsbad, CA, USA) according to manufacturer instructions. In each well, $4 \mu \mathrm{g}$ pSEH-Flag-HBV was transfected. Cells were chosen at $48 \mathrm{~h}$ post-transfection with $450 \mu \mathrm{g} / \mathrm{mL}$ hygromycin. Cell colonies were obtained after approximately 14 days of screening and transferred to 96-well plates in the inverted microscope and enlarged in a $35-\mathrm{cm}^{2}$ culture flask.

\section{Detection of hepatitis B e antigen (HBeAg) and hepatitis B s antigen (HBsAg) expression}

All cell colonies were seeded in $50 \%$ cell confluences on a $6-\mathrm{cm}$ dish. Conditioned medium from each cell line was assayed at $450 \mathrm{~nm}$ according to supplier recommendations (Shanghai Kehua Bio-Engineering Enzyme Immunoassay kit for hepatitis B e/core [HBe] antigen and surface [HBs] antigen) after $48 \mathrm{~h}$. The absorbance of samples at $450 \mathrm{~nm}$ was measured with a Synergy HT Multi-Detection Microplate Reader (BioTeK). Optical density values greater than 2.1-fold the negative control were considered positive. Each sample was repeated in 3 independent experiments.

\section{DNA preparations and polymerase chain reaction (PCR) assay}

Cellular genomic DNA was prepared from $10 \times 10^{6}$ cells suspended in $1 \mathrm{~mL}$ lysis buffer $(10 \mathrm{mM}$ Tris- $\mathrm{HCl}, \mathrm{pH} 7.8,5 \mathrm{M}$ ethylenediaminetetraacetic acid, $0.5 \%$ sodium dodecyl sulfate), lysed with $20 \mu \mathrm{L}$ proteinase $\mathrm{K}\left(20 \mu \mathrm{g} / \mathrm{mL}\right.$; Promega), and incubated at $55^{\circ} \mathrm{C}$ for $2 \mathrm{~h}$. The DNA was then treated with phenol/chloroform/isoamyl alcohol $(25: 24: 1, \mathrm{v} / \mathrm{v} / \mathrm{v})$, precipitated with $75 \%$ ethanol, and dissolved in $50 \mu \mathrm{L}$ double-distilled water. The primers were designed on HBx and hygromycin genes, which amplified fragment lengths of 462 and $347 \mathrm{bp}$, respectively. The primers for X gene are: HBx-F, 5'-ATGGCTGCTAGGCTGTACTG-3'; HBx-R, 5'-GGCA GAGGTGAAAAAGTTGCA-3') and the primers for hygromycin gene are hygromycin-F, 5'-TTTCGGCTCCAACAATGTCC-3'; hygromycin-R, 5'-AGCTGCATCATCGAAATTGC $\left.-3^{\prime}\right)$. PCR was performed under the following conditions: an initial step of $5 \mathrm{~min}$ at $95^{\circ} \mathrm{C}$, a differential cycle of $45 \mathrm{~s}$ at $95^{\circ} \mathrm{C}, 60 \mathrm{~s}$ at $55^{\circ} \mathrm{C}$, and $60 \mathrm{~s}$ at $72^{\circ} \mathrm{C}$, followed by a step at $72^{\circ} \mathrm{C}$ for 5 min for a total of 30 cycles.

\section{RNA preparation and reverse transcriptase PCR assay}

Total RNA was isolated using TRIzol according to manufacturer instructions and was treated using a trace DNA Removal Kit (Promega) to eliminate residual DNA. Concentration and purity were then determined spectrophotometrically. The same amount of RNA was reverse transcribed to obtain complementary DNA using a Reverse Transcription System (Promega) according to manufacturer instructions. Primers, positive controls, and reaction conditions were consistent with conventional PCR conditions.

\section{Southern blot analysis of the integration of HBV DNA into the cellular genome}

DNA was extracted from the stable cell line and digested with 4 restriction enzymes. All 
purified DNA samples were electrophoresed through 1.0\% agarose gel and transferred to a nylon membrane. Hybridization was performed overnight at $42^{\circ} \mathrm{C}$ with 1.1 -fold full-length HBV DNA with digoxigenin-labeled nick-translation. A 3.5-kb HBV fragment was used as a positive control.

\section{RESULTS}

\section{Characterization of the recombinant vector}

Reconstructed pSEH-Flag-HBV plasmids (10.9 kb) were run as 2 fragments after HindIII digestion (Figure 1), which yielded the expected results (6.7 and $4.2 \mathrm{~kb}$ ). Sequencing results verified that the recombinant plasmid had been successfully constructed (data not shown).

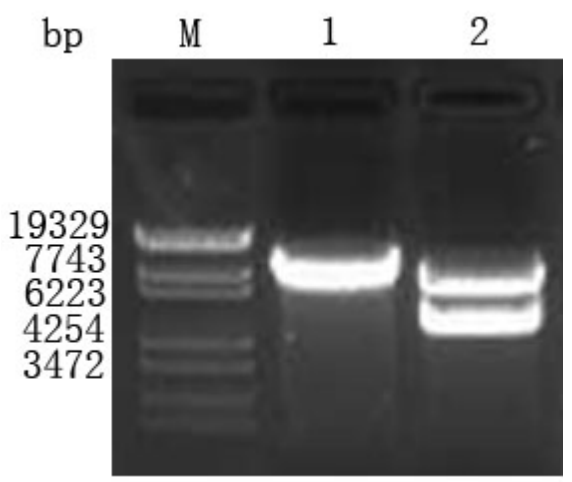

Figure 1. Identification of pSEH-Flag-HBV plasmid by single digestion of HindIII. Lane $M=\lambda$-EcoT14 I digest DNA marker; lane 1 = pSEH-Flag-HBV plasmid; lane 2 = pSEH-Flag.

\section{HBeAg and HBsAg screening of monoclonal cell using enzyme-linked immunosorbent assay}

pSEH-Flag-HBV plasmids were transfected into HepG2 cells and screened with 450 $\mu \mathrm{g} / \mathrm{mL}$ hygromycin for 14 days. Thirty-seven hygromycin-positive colonies were formed. The conditioned medium of each clone was collected and assayed for HBsAg and HBeAg expression. We found that the HepG2-H4 cell line produced HBsAg and HBeAg stably, and compared with the HepG2.2.15 cell line, HepG2-H4 produced more negative and positive HBsAg and $\mathrm{HBeAg}$ (Figure 2).

\section{Identification of HBV DNA and RNA in HepG2-H4 cells}

To demonstrate that the HepG2-H4 cell line can stably express HBsAg and $\mathrm{HBeAg}$, we checked for the existence of the HBV genome in HepG2-H4 cellular DNA using PCR. We found that both HBV and hygromycin could be amplified with HepG2-H4 cellular DNA (Figure 3A). Furthermore, HBV mRNA expression was identified using reverse transcriptase PCR. Both HBV and hygromycin were expressed in HepG2-H4 cells (Figure 3B). 


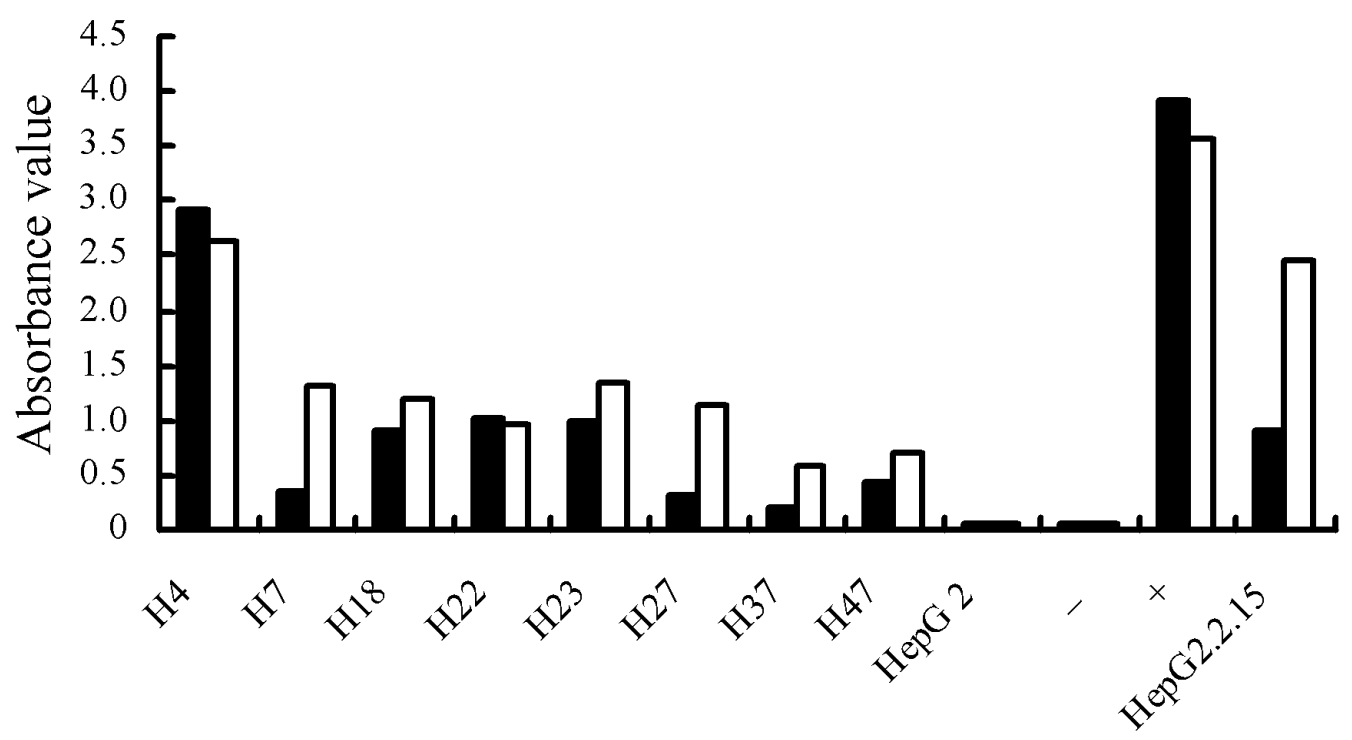

Figure 2. Absorption values of HBsAg (filled columns) and HBeAg (open columns) were detected by ELISA. H4, $\mathrm{H} 7, \mathrm{H} 18, \mathrm{H} 22, \mathrm{H} 23, \mathrm{H} 27, \mathrm{H} 37$, and H47 are cell line numbers. Values shown are mean of $\mathrm{N}=3$, and each sample was performed in triplicate. $(-)=$ Negative control; $(+)=$ positive control.
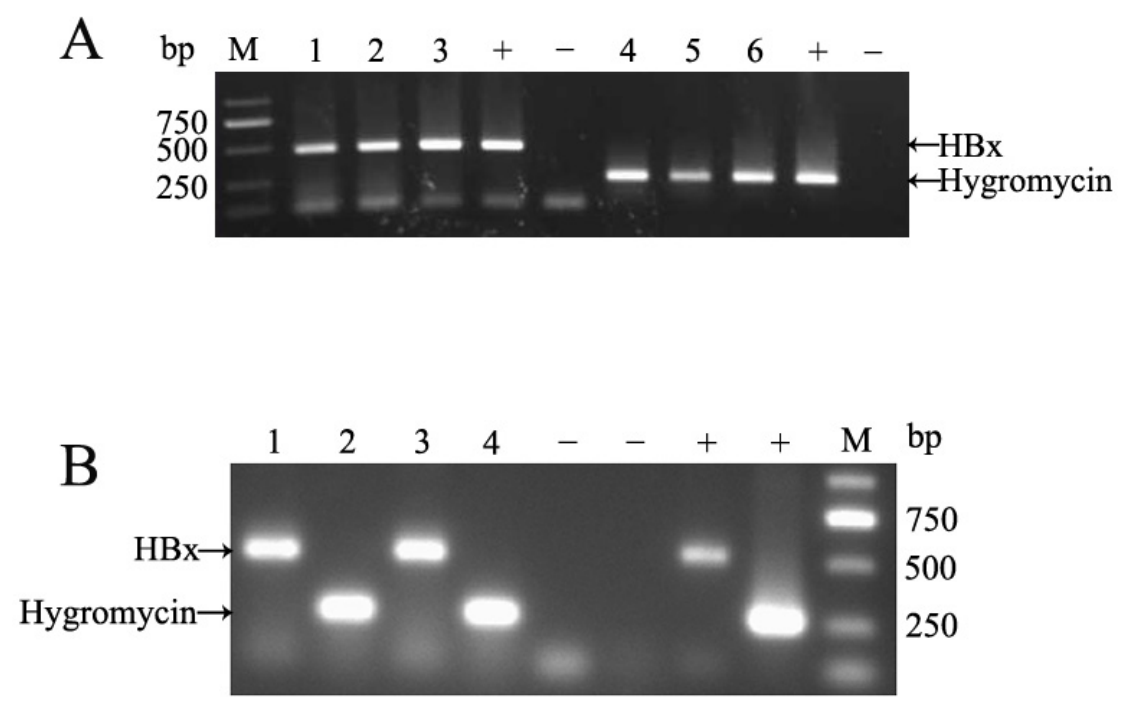

Figure 3. Identification of HBV DNA and RNA in HepG2-H4 cells. A. HBV, Hygromycin gene have been integrated in HepG2-H4 cellular DNA. H4, H22, H23 cells were amplified with X, H primer. Lane M= DL2000 DNA marker; lanes 1-3 = H4, H22, H23 cells were amplified with X primer; lanes 4- $6=\mathrm{H} 4, \mathrm{H} 22, \mathrm{H} 23$ cells were amplified with Hygromycin primer; lanes $+=$ positive control; lanes $-=$ negative control. B. The expressions of $\mathrm{HBx}$ and Hygromycin mRNA were confirmed by RT-PCR. Lanes 1 and $3=\mathrm{H} 4$, H22 cells amplified with X primer; lanes 2 and $4=\mathrm{H} 4, \mathrm{H} 22$ cells amplified with hygromycin primer; lanes $-=\mathrm{HepG} 2$ transfected with control plasmid used as negative control; lanes $+=$ pSEH-Flag-HBV plasmid used as positive control; lane $M=$ DL2000 DNA marker. 


\section{Identification of integration of HBV DNA}

To determine the number of copies of HBV DNA integrated into HepG2-H4 chromosomes, total HepG2-H4 cellular DNA was isolated from the lysed cells for Southern blot analysis. As shown in Figure 4, the samples analyzed were digested with BglII (lane 1), EcoRI (lane 2), SphI (lane 3), and SacI (lane 4). A 3.5-kb fragment of HBV was used as a probe to analyze HBV in HepG2-H4 chromosomes. We determined that the HBV genome was integrated singly into the HepG2-H4 cell line (see Figure 4).

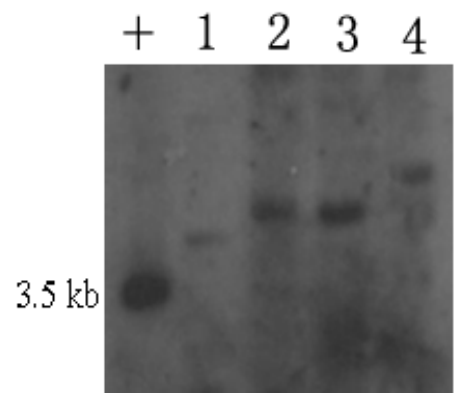

Figure 4. Southern blot analysis for integration of HBV genome in HepG2-H4 intracellular DNA. HepG2-H4 genomic DNA were digested with BgIII (lane 1), EcoRI (lane 2), SphI (lane 3), SacI (lane 4). HBV fragment (3.5 $\mathrm{kb}$ ) was used as probe to detect existence of HBV DNA. Lane $+=3.5-\mathrm{kb}$ HBV fragment used as a positive control.

\section{DISCUSSION}

With the advent of molecular biology, inserting recombinant genes into human hepatocellular carcinoma cells has become a powerful tool for the identification and analysis of the expression of functional gene products. This technique has greatly accelerated the study of HBV. We successfully constructed a hygromycin-resistant cell line stably expressing HBV to ensure genomic continuity after chromosomal integration and the efficient transcription of viral RNA. We have shown herein that a mere 1.3-fold HBV genome is large enough to act as a template for the synthesis of all HBV mRNAs. In the HepG2-H4 cell line, HBsAg and HBeAg can be highly expressed owing to the strong foreign hEFH (Human EF1 $\alpha /$ HLTV hybrid) promoter of HBV, which comes from the pSEH-Flag plasmid.

HepG2 cells have been shown to maintain a substantial number of liver-specific functions (Knowles et al., 1980). The viral genome integrated just 1 copy into HepG2 cell chromosomes in our HepG2-H4 cells, implying that the extraneous HBV DNA integration affects just 1 gene in HepG2-H4 cells, unlike that in HepG2.2.15 cells, in which HBV is integrated into 4 sites in HepG2.2.15 cellular DNA (Sells et al., 1988). This integration reduces damage to the HepG2 cells by foreign HBV DNA. We intend to study the position of HBV integration into the host genome.

HepG2-H4 cells can be used to test the effects of drugs that could interfere with viral replication. These cells can also serve as an in vitro model for the analysis of gene involvement in HBV disease processes. Thus, compared with other HBV-producing cell lines established thus far, the features of the HepG2-H4 system make it a more effective candidate with which to study HBV. 


\section{ACKNOWLEDGMENT}

Research supported by the Nature Science Foundation of China (\#30771924) and the Natural Science Foundation Project of CQ CSTC (\#2010BB5359).

\section{REFERENCES}

Ganem D, Weiser B, Barchuk A, Brown RJ, et al. (1982). Biological characterization of acute infection with ground squirrel hepatitis virus. $J$. Virol. 44: 366-373.

Halpern MS, England JM, Flores L, Egan J, et al. (1984). Individual cells in tissues of DHBV-infected ducks express antigens crossreactive with those on virus surface antigen particles and immature viral cores. Virology 137: 408-413.

Knowles BB, Howe CC and Aden DP (1980). Human hepatocellular carcinoma cell lines secrete the major plasma proteins and hepatitis B surface antigen. Science 209: 497-499.

Lee W (1997). Hepatitis B virus infection. N. Engl. J. Med. 337: 1733-1745.

Lindberg J, Pichoud C, Hantz O, Vitvitski L, et al. (1985). Woodchuck hepatitis virus infection: serologic and histopathologic course and outcome. Eur. J. Clin. Microbiol. 4: 59-61.

Ochiya T, Tsurimoto T, Ueda K, Okubo K, et al. (1989). An in vitro system for infection with hepatitis B virus that uses primary human fetal hepatocytes. Proc. Natl. Acad. Sci. U. S. A. 86: 1875-1879.

Sells MA, Chen ML and Acs G (1987). Production of hepatitis B virus particles in Hep G2 cells transfected with cloned hepatitis B virus DNA. Proc. Natl. Acad. Sci. U. S. A. 84: 1005-1009.

Sells MA, Zelent AZ, Shvartsman M and Acs G (1988). Replicative intermediates of hepatitis B virus in HepG2 cells that produce infectious virions. J. Virol. 62: 2836-2844.

Weiss L, Kekule AS, Jakubowski U, Burgelt E, et al. (1996). The HBV-producing cell line HepG2-4A5: a new in vitro system for studying the regulation of HBV replication and for screening anti-hepatitis B virus drugs. Virology 216: 214-218.

Will H, Cattaneo R, Koch HG, Darai G, et al. (1982). Cloned HBV DNA causes hepatitis in chimpanzees. Nature 299: 740-742. 